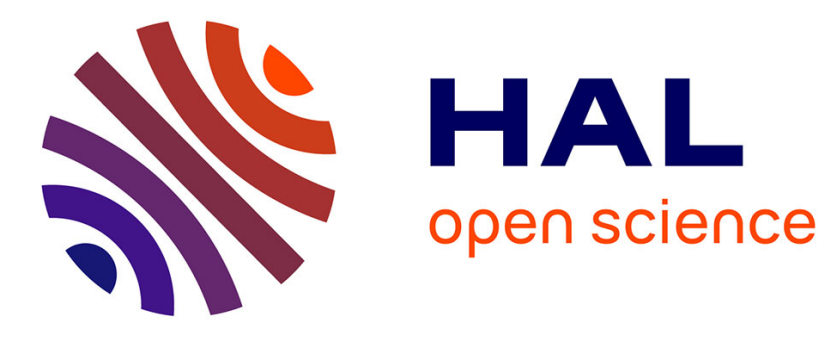

\title{
Golden Ratio Geometry and the Fine-Structure Constant
}

Michael Sherbon

\section{To cite this version:}

Michael Sherbon. Golden Ratio Geometry and the Fine-Structure Constant. Journal of Advances in Physics, 2019, 16 (1), pp.362-368. 10.24297/jap.v16i1.8469 . hal-02341850

\section{HAL Id: hal-02341850 https://hal.science/hal-02341850}

Submitted on 31 Oct 2019

HAL is a multi-disciplinary open access archive for the deposit and dissemination of scientific research documents, whether they are published or not. The documents may come from teaching and research institutions in France or abroad, or from public or private research centers.
L'archive ouverte pluridisciplinaire HAL, est destinée au dépôt et à la diffusion de documents scientifiques de niveau recherche, publiés ou non, émanant des établissements d'enseignement et de recherche français ou étrangers, des laboratoires publics ou privés. 


\title{
Golden Ratio Geometry and the Fine-Structure Constant
}

\author{
Michael A. Sherbon \\ Case Western Reserve University Alumnus, United States \\ E-mail: michael.sherbon@case.edu
}

October 4, 2019

\begin{abstract}
The golden ratio is found to be related to the fine-structure constant, which determines the strength of the electromagnetic interaction. The golden ratio and classical harmonic proportions with quartic equations give an approximate value for the inverse fine-structure constant the same as that discovered previously in the geometry of the hydrogen atom. With the former golden ratio results, relationships are also shown between the four fundamental forces of nature: electromagnetism, the weak force, the strong force and the force of gravitation.
\end{abstract}

Keywords fine-structure constant, fundamental constants, history of physics, history of mathematics, golden ratio, quartic equation.

\section{Introduction}

From John Rigden, "The fine-structure constant derives its name from its origin. It first appeared in Sommerfeld's work to explain the fine details of the hydrogen spectrum. ... Since Sommerfeld expressed the energy states of the hydrogen atom in terms of the constant [alpha], it came to be called the fine-structure constant." [1]. Arnold Sommerfeld states, "In our theory of the fine structure there is a confluence of the three main currents of modern research in theoretical physics, namely, the theory of electrons, the theory of quanta, and the theory of relativity" and the fine-structure constant " ... could be interpreted more physically as the ratio of an electron's velocity in the first Bohr orbit to the speed of light." [2]. From previous work, "The electromagnetic coupling constant determining the strength of its interaction is the fine-structure constant $\alpha=e^{2} / \hbar c$ in $\operatorname{cgs}$ units with the elementary charge $e$, the reduced Planck's constant $\hbar=h / 2 \pi$ and the speed of light $c . "$ [3].

The fine-structure constant has a variety of physical interpretations from which measurements and determinations have been made. Even though the fine-structure constant is often defined as 
a low energy limit, in high energy physics it has become commonplace to regard it of less importance because the gauge coupling runs with energy. As Toichiro Kinoshita explains, “... the detailed high energy structure of the ultimate theory is irrelevant to the analysis of low energy phenomena except insofar as it determines these parameters." [4]. What actually determines the fine-structure constant remains questionable for high energy theorists.

A reminder from Jesse DuMond's work that is still of significance today:

These results illustrate clearly the highly interrelated nature of the fundamental constants. Dependent as our knowledge of them is upon many different fields of physics, we have here a good example of the importance of making occasional analyses of the consistency situations of sufficiently inclusive scope to serve as valuable guides to further research. The present example emphasizes especially that a better knowledge of the Sommerfeld constant, $\alpha$, would be of great value to physics at the present time [5].

From the writings of Wolfgang Pauli, "The theoretical determination of the fine structure constant is certainly the most important of the unsolved problems of modern physics." [6, 7]. Richard Feynman said it was the greatest mystery of physics, perhaps the most often quoted physicist on the fine-structure constant: "It has been a mystery ever since it was discovered more than fifty years ago, and all good theoretical physicists put this number up on their wall and worry about it. ... You might say the 'hand of God' wrote that number, and 'we don't know how He pushed his pencil"” [8].

In the Timaeus, Plato "... considered the golden section to be the most binding of all mathematical relationships and the key to the physics of the cosmos," as quoted by Robert Schoch and Robert McNally in Pyramid Quest [9]. Peter Champoux and William Buehler state that, "The capstone of the Pyramid generates the golden spirals which creates the whole form." [10].

The golden ratio $\phi=(1+\sqrt{5}) / 2$ and is a root of its minimal polynomial $x^{2}-x-1$. Both the golden ratio and its conjugate are roots of the quartic equation $x^{4}-2 x^{3}-x^{2}+2 x+1=0$. For "... a particular quartic plane curve, different combinations of the coefficients of the general curve give rise to the lemniscate of Bernoulli. ... Gauss's and Euler's study of the arc length of Bernoulli's lemniscate, a polar curve having the general form of a toric section, led to later work on elliptic functions." [11]. The inflection points of the quartic are also related to the golden ratio [12].

\section{Calculation of the fine-structure constant}

An approximation from previous work [3], the inverse fine-structure constant $\alpha^{-1}$ is a root of:

$$
x^{4}-137 x^{3}-10 x^{2}+697 x-365=0 .
$$

This equation gives a value of for $x$ as $\alpha^{-1} \simeq 137.035999168$. The latest determination from the Gabrielse group [13] is $\alpha^{-1} \simeq 137.035999150$ (33), from experimental measurement and quantum electrodynamics [14]. This calculation of the inverse fine-structure constant gives the same approximate value as ancient geometry combined with the extension of Raji Heyrovska's 
work on the golden ratio structure of the hydrogen atom [15]. $697-137=280+280.365+$ $365-10=280+440=720$.

Also stated previously, "The scaling factor for the second pyramid on the Giza Plateau is 137, having a height of $2 \times 137=274$ Royal Cubits and a base of $3 \times 137=411$ Royal Cubits." [3]. "The Great Pyramid has a combined height and base of $280+440=720$ Royal Cubits, $280 / 220=14 / 11 \simeq \sqrt{\phi}$ and 220/136 $\simeq \phi . "$ [3]. Colonel R. S. Beard states that, "Sir William Petrie himself was thoroughly convinced that the Egyptians constructed the pyramid with a height-to-width-of-base ratio of seven to eleven." [16].

Ernest Pecci quotes Thoth: "The Pyramid is a representation of all created things. All matter consists of cosmic frequencies held in place by the angulations contained within specific harmonic triangles that are found within the Platonic solids. ... the principle of proportion determines and maintains all forms." [17].

Another approximation from our previous work [3] also gives the same approximate value for the inverse fine-structure constant. $\alpha^{-1} \simeq \phi x$, where $x$ is a root of:

$$
3 x^{4}-250 x^{3}-346 x^{2}+48 x-36=0 .
$$

$280=36-3-3+250,440=36-346+(3 \times 250)$ and $346=48+48+250$. Another quartic equation having the Eq. (1) value with $\alpha^{-1} \simeq \pi^{7} / 7 \pi \sqrt{x}$, where $x \simeq 4 / \pi \sqrt{\phi} \simeq 1$ is a root of:

$$
138 x^{4}+83 x^{3}-70 x^{2}+7 x-161=0 .
$$

$137=7+70+83+138-161,280=83+83+137+138-161$ and $440=7+70+83+280$. $\pi^{7} / 7 \pi$ is from the Hebrew inverted heart Tetractys or Tetragrammaton from 'Tengri 137' [18]. The $\pi^{7}$ is the perimeter of the Great Pyramid base and $7 \pi$ is found in the Golden Apex of the Great Pyramid, see Eq. (9) discussion. Jerry Gin states,

It is also interesting to note that the name of God in Hebrew is YHWH (or Yahweh) which is translated as 'Tetragrammaton' from Greek. Tetra refers to four and may refer to the four faces of a tetrahedron and grammaton may refer to weight or gravity [19].

Ernest Pecci explains, "One importance of the Tetractys lies in its expansion to 3-dimensional and multi-dimensional levels. The Star of David, The Tree of Life, and the Tetragrammaton of the heart are all based upon the simple equilateral triangle divided into the ten dots of the Tetractys." [20]. Gordon Plummer says, "Perhaps of paramount importance is that of the Tetraktys. This was the symbol probably most revered by the Pythagoreans." [21, 22].

For Manly P. Hall: "The tetrad -4- was esteemed by the Pythagoreans as the primogenial number, the root of all things, the fountain of nature and the most perfect number. ... Wherein the Pythagoreans expressed God as a tetrad is explained in a sacred discourse ascribed to Pythagoras, wherein God is called the Number of Numbers." [23].

Leon Marvell writing about Robert Fludd: "Interestingly, Fludd's conviction that the Tetragrammaton or deity was the infinite dimension within and between all things is an early precursor of late nineteenth-century conceptions (such as those of William Clifford) that saw the fourth dimension as precipitating phenomena such as the appearance of matter." [24]. 
Another quartic polynomial also gives the same approximate value for $\alpha^{-1}$. The inverse finestructure constant $\alpha^{-1} \simeq 4 R \sinh (x)$, with $R$ as the Reality Number from the Great Pyramid [25] and $x$ is a root of:

$$
38 x^{4}+313 x^{3}-273 x^{2}-44 x+50=0 .
$$

This quartic has a root $x \simeq \phi / e$, with the golden ratio and Euler's number. $4=44+273-$ $313,280=4(38+38+44-50), 160=4(313-273)$, and $440=160+280$. Also, $\alpha^{-1} \simeq$ $R \cosh ^{-1}(2 \pi) \simeq R \sqrt{2 \pi}$, see the reference to Alfred Landé in our previous work [3]. The Reality Number, $R$, “. $\ldots$ is an angle by which the least amount of energy is needed to accomplish the most amount of good when rotating from one dimension into the next." [25].

$R=54.277$ degrees. This value is encoded into the structure in this way: since the Pyramid has the apothem angle of 51.853... degrees, and since the Ascending and Descending Passages needed a specified angle between them ... the value exactly midway between the apothem angle and the $R$ number was chosen. Step one is from $51.853 \ldots$ degrees to 53.065 degrees. Step two is from 53.065 degrees to 54.277 degrees. ... and the three angles honor the ascension principle [25].

$R \simeq 7 A B$, where $A$ is the Golden Apex of the Great Pyramid and $B=\tan ^{-1}(4 / \pi)$ is the base angle of the Great Pyramid [26]. $\theta_{g} \simeq 26.57^{\circ} \simeq \tan ^{-1}(1 / 2)$ is the ancient Golden Angle of Resurrection noted by Robert Temple [27]. $\theta_{g} \simeq(R+B) / 4$ and $7=\phi^{4}+\phi^{-4} \simeq \sinh \sqrt{7}$. Another approximation for the inverse fine-structure constant $\alpha^{-1} \simeq \exp (4 x)$, where $x \approx 2 / \phi$ is a root of:

$$
7 x^{4}-28 x^{3}+9 x^{2}-11 x+36=0 .
$$

This equation also gives the same approximate value for the inverse fine-structure constant as Eq. (1). $280=28(11+36-9-28) .440=(9+11)(11+11)$. The inverse fine-structure constant $\alpha^{-1} \simeq(4 \times 4) x$, where $x \simeq 5 \phi^{4} / 4$ is a root of:

$$
2 x^{4}+44 x^{3}-550 x^{2}+233 x-56=0 .
$$

This equation also gives the same approximate value for the inverse fine-structure constant as Eq. (1). $280=550-56-56+2$ and $440=44(56-44-2)=2(44-56+233)-2$. The inverse fine-structure constant $\alpha^{-1} \simeq 372 / x$, where $x \simeq e \simeq \phi \sqrt{2 \sqrt{2}}$ :

$$
16 x^{4}+25 x^{3}-37 x^{2}-97 x-833=0 .
$$

$4 \times 4=16$ is the main harmonic number of the Great Pyramid according to Karim [28] and Witteveen [29]. Karim states that,

The proportion derived from that number (1:1.6) is the famous Golden Proportion used in Ancient architecture and now the basis of 'Sacred Geometry'. Sixteen brings out the sacred quality hidden within the One to manifest in our time-space dimension [28].

The $\ln 372 \simeq 1+\ln 137$ and $\sinh ^{-1}(372) \simeq A^{-1}$, inverse Golden Apex of the Great Pyramid [30]. The harmonic sum of 372 is part of a spectrum analysis related to crystallographic groups [30]. 
$37 / 7 \pi \simeq \sqrt{2 \sqrt{2}} \simeq 1+\sin \alpha^{-1}$ and $\phi / \ln \phi \simeq 2 \sqrt{2 \sqrt{2}} .280=16(37-25)+97-25+16$ and $440=16+97+97-25-25+280.440+440=97-25-25+833$.

From Robert Lawlor describing harmonic division with the square root of two, "Here is a means of drawing a beautifully proportioned cup or grail-shaped vessel, using only the harmonic division to establish its curves and measures. We may speculate that this is the geometric essence of the Holy Grail." [31].

This grail geometry includes $\sqrt{2}-1 \simeq \phi^{2} / 2 \pi$ and $2-\sqrt{2} \simeq 4 / \phi^{4} \simeq 4 A$. Also, in radians $\cot (\sqrt{2}-1) \simeq 1+\sqrt{\phi}$, linked to Plato's beautiful scalene triangle found in the Cosmological Circle extension known as Plato's Wheel [20]. Ernest Pecci says:

It is of significance that $\sqrt{2}-1,2-\sqrt{2}, 1$ creates a 3 -term harmonic proportion that places the baseline of the Pyramid at the harmonic mean between the floor of the Subterranean Chamber and the floor of the King's Chamber! [20].

From the Pythagorean perspective the Foundation Stone number of 729 is a harmonic of the fine-structure constant value. Another equation from previous work [3] that also gives the same approximate value for the inverse fine-structure constant as Eq. (1) is $\alpha^{-1} \simeq 729 / x$, where $x$ is a root of:

$$
10 x^{4}-37 x^{3}-113 x^{2}+73 x+371=0 .
$$

The root $x \simeq \phi \phi_{f}$, where $\phi_{f}$ is the reciprocal Fibonacci constant. $280=10+10-37-73+$ $(10 \times 37)$ and $440=73+371-((113-73) / 10)$.

Czech physicist Raji Heyrovska says, "On noticing the closeness of the fine structure constant $\ldots$ to the ratio of the angles, $360^{\circ} / \phi^{2} \ldots$ the author suggested that the small difference ... could be due to the Sommerfeld's relativity correction factor." [33]. "It was also pointed out that the ratio $360^{\circ} / \phi^{2} \ldots$ which is a Golden section of $360^{\circ}$, differs from the inverse fine structure constant by $\ldots 2 / \phi^{3} \ldots$ probably due to the difference in the g-factors for the electron and proton ...," with the result of $\alpha^{-1} \simeq\left(360 / \phi^{2}\right)-\left(2 / \phi^{3}\right)$ [34].

$$
\alpha^{-1} \simeq \frac{360}{\phi^{2}}-\frac{2}{\phi^{3}}+\frac{A^{2}}{K \phi^{4}}-\frac{A^{3}}{K^{2} \phi^{5}}+\frac{A^{4}}{K^{3} \phi^{7}} .
$$

This extension of Heyrovska's equation found previously also gives $\alpha^{-1} \simeq 137.035999168$ [14]. Raji Heyrovska found it a

... surprise to find for the first time that the Bohr radius is divided into two unique sections at the point of electrical neutrality, which is the Golden point. The Golden ratio, which manifests itself in many spontaneous creations of Nature, was thus found to originate right in the core of atoms [34].

William Eisen describes the geometry of what he called the 'Golden Apex of the Great Pyramid' where dividing the sides of his mathematical model for the Great Pyramid by $\pi$ lengths along with four curves of the exponential function results in a small square in the center called the Golden Apex, the geometry and symmetry thought to be associated with the generation of the four fundamental forces of nature [30]. The Golden Apex $A$ is the side length of the resulting square. $A=e^{\pi}-7 \pi-1 \simeq \sqrt{\pi \alpha} \simeq \pi / \sqrt{440} \simeq 3 / 20$. 
The inverse Kepler-Bouwkamp constant is the polygon circumscribing constant $K \simeq \phi^{2} / 2 A \simeq$ 8.7 and $\sin \alpha^{-1} \simeq 7 ! /(713+137) K \simeq(\pi-1) / \pi$ [11]. Also of interest, $\ln (\phi / \alpha) \simeq \phi / 2 A \simeq K / \phi$ and $11 / 37 \simeq \cos \sqrt{\phi} \simeq 2 A$ [3]. The polygon circumscribing constant "has interesting geometric connections with the torus topology relating the relativity of Einstein with the geometry of classical and quantum mechanics." [7]. The Reality Number $R \simeq 7 A B \simeq 2 \pi K \simeq 360 A$, see Eq. (4) discussion.

Approximations from previous work [30] involving the four fundamental forces:

$$
A \simeq 4 \pi \phi \alpha \simeq \alpha_{w} / \sin ^{2} \theta_{w} \simeq \pi \phi \alpha_{s} / 4 \simeq K \sqrt{\pi} / \ln \alpha_{G}^{-1} .
$$

With $\sin ^{2} \theta_{w}$ as the Weinberg angle or the weak mixing angle, $\alpha_{w}$ is the weak nuclear force, $\alpha_{s}$ is the strong nuclear force and $\alpha_{G}$ is the gravitational coupling constant [35]. The inclusion of the four fundamental forces in the Golden Apex design is part of the symbolic interpretation presented by William Eisen [36]. The geometry of the Tetraktys has been associated with the four elements and the four fundamental forces.

\section{Conclusion}

The inverse fine-structure constant calculated with quartic polynomials and the main harmonic parameters of the Great Pyramid have suggested the golden ratio as the mathematical basis for the fine-structure constant. From previous work “... several more formulations for the finestructure constant with the same approximate value have connections with prime number theory, the real fixed point of the hyperbolic cotangent, anomalous magnetic moment of the electron, Laplace limit of Kepler's equation and harmonic proportions of the Cosmological Circle" [3, 30, 37].

\section{Acknowledgments}

Special thanks to Case Western Reserve University, MathWorld and WolframAlpha.

\section{References}

[1] Rigden, J.S. Hydrogen: The Essential Element, Cambridge, MA: Harvard University Press, 2003, 55 .

[2] Sommerfeld, A. Atomic Structure and Spectral Lines, New York, NY: Dutton, 1934.

[3] Sherbon, M.A. "Fine-structure Constant from Sommerfeld to Feynman," Journal of Advances in Physics, 16, 1, 335-343 (2019).

[4] Kinoshita, T. Quantum Electrodynamics, Singapore: World Scientific, 1990, 3.

[5] DuMond, J.W.M. "The Present Key Importance of the Fine Structure Constant, $\alpha$, to a Better Knowledge of All the Fundamental Physical Constants," Journal for Nature Research, A 21, 1-2, 70-79 (1966). 
[6] Pauli, W., Enz, C.P. \& Meyenn, K. Writings on Physics and Philosophy, Berlin: Springer, 1994.

[7] Sherbon, M.A. "Wolfgang Pauli and the Fine-Structure Constant," Journal of Science, 2, 3, 148-154 (2012).

[8] Feynman, R.P. QED: The Strange Theory of Light and Matter, Princeton, NJ: Princeton University Press, 1985, 129.

[9] Schoch, R.M. \& McNally, R.A. Pyramid Quest: Secrets of the Great Pyramid and the Dawn of Civilization, New York, NY: Jeremy P. Tarcher/Penguin, 2005, 168.

[10] Champoux, P.W. \& Buehler, W.S. Gaia Matrix: Arkhom and the Geometries of Destiny in the North American Landscape, Washington, MA: Franklin Media, 1999, 200.

[11] Sherbon, M.A. "Fundamental Nature of the Fine-Structure Constant," International Journal of Physical Research, 2, 1, 1-9 (2014).

[12] Totland, H. "Quartic Polynomials and the Golden Ratio," Mathematics Magazine, 82, 3, 197-201 (2009).

[13] Gabrielse, G., Fayer, S.E., Myers, T.G. \& Fan, X. "Towards an Improved Test of the Standard Model's Most Precise Prediction," Atoms, 7, 2, 45 (2019).

[14] Aoyama, T., Kinoshita, T. \& Nio, M. "Theory of the Anomalous Magnetic Moment of the Electron," Atoms, 7, 1, 28 (2019).

[15] Sherbon, M.A. "Fine-Structure Constant from Golden Ratio Geometry," International Journal of Mathematics and Physical Sciences Research, 5, 2, 89-100 (2018).

[16] Beard, R.S. "The Fibonacci Drawing Board Design of the Great Pyramid of Gizeh," The Fibonacci Quarterly, 6, 85-87 (1968).

[17] Pecci, E.F. The Great Pyramid: A Temple of Created Life, Walnut Creek, CA: Pavior Publishing, 2014, 3.

[18] Anon. “Tengri 137," Wikia. https://tengri137.fandom.com/wiki/Tengri_137_Translation (2016).

[19] Gin, J. "Fundamental Pattern and Consciousness," Cosmos and History, 12, 2, 99-113 (2016).

[20] Pecci, E.F. The Sacred Geometry of the Great Pyramid, Walnut Creek, CA: Pavior Publishing, 2010, 28.

[21] Plummer, L.G. The Mathematics of the Cosmic Mind: A Study in Mathematical Symbolism, Wheaton, IL: Theosophical Publishing House, 1970, 17.

[22] Plummer, L.G. By the Holy Tetraktys!: Symbol and Reality in Man and Nature, San Diego, CA: Point Loma Publications, 1982. 
[23] Hall, M.P. The Secret Teachings of All Ages, Mineola, NY: Dover Publications, 2010, 171.

[24] Marvell, L. The Physics of Transfigured Light: The Imaginal Realm and the Hermetic Foundations of Science, Rochester, VT: Inner Traditions, 2016, 231-232.

[25] Miller, R.G. Pyramid Truth Gateway Universe, Blue Hill, ME: Medicine Bear Publishing, 1997, 201, 222.

[26] Sherbon, M.A. "Quintessential Nature of the Fine-Structure Constant," Global Journal of Science Frontier Research, A, 15, 4, 23-26 (2015).

[27] Temple, R.G. Egyptian Dawn: Exposing the Real Truth Behind Ancient Egypt, London, UK: Century, 2010.

[28] Karim I. Back to a Future for Mankind: BioGeometry, Montreal, CA: BioGeometry Consulting, 2007, 194.

[29] Witteveen, W. The Great Pyramid of Giza: A Modern View on Ancient Knowledge, Kempton, IL: Adventures Unlimited Press, 2016, 347.

[30] Sherbon, M.A. "Fundamental Physics and the Fine-Structure Constant," International Journal of Physical Research, 5, 2, 46-48 (2017).

[31] Lawlor, R. Sacred Geometry: Philosophy and Practice, London, UK: Thames and Hudson, $1982,85$.

[32] Eisen, W. "The Mysteries of the Constants (e) and (i)," in The Universal Language of Cabalah, Marina Del Rey, CA: DeVorss, 1989, 160-178.

[33] Heyrovska, R. "The Golden Ratio, Ionic and Atomic Radii and Bond Lengths," Molecular Physics, 103, 877 - 882 (2005).

[34] Heyrovska, R. "Golden Ratio Based Fine Structure Constant and Rydberg Constant for Hydrogen Spectra," International Journal of Sciences, 2, 5, 28-31 (2013).

[35] Kunes, J. Dimensionless Physical Quantities in Science and Engineering, London, UK: Elsevier, 2012, 15.

[36] Eisen, W. The English Cabalah Volume II: The Mysteries of Phi, Marina Del Rey, CA: DeVorss, 1982.

[37] Sherbon, M.A. "Physical Mathematics and The Fine-Structure Constant," Journal of Advances in Physics, 14, 3, 5758-64 (2018). 